\title{
Alimentação saudável na experiência de idosos
}

Healthy alimentation on the experience of the elderly

Resumo

Procuramos, neste artigo, identificar o que significa alimentação saudável para pessoas idosas e dificuldades encontradas no cotidiano para a incorporação desse conjunto de preceitos. Entrevistamos 202 idosos com mais de 60 anos, a maioria do sexo feminino $(93,1 \%)$ e frequentadores atuais ou pregressos de atividades da Universidade Aberta da Terceira Idade da Universidade do Estado do Rio de Janeiro $(70,8 \%)$. As respostas indicam um ideário que apresenta estreita vinculação à preocupação com a saúde, no sentido de prevenir ou tratar as doenças crônicodegenerativas, com caráter biologicista e medicalizador do ato de comer distanciado do mundo dos desejos e da subjetividade. Dificuldades relativas a poder aquisitivo, vida em família ou na solidão, questões biológicas impostas pelo processo de envelhecimento aparecem como limitantes para a prática regular da alimentação saudável. Acreditamos que, a partir de reflexões sobre essas questões, seja possível enriquecer a noção de alimentação saudável, incluindo efetivamente em seu interior tanto a saúde, quanto a doença, considerando a importância de um diálogo que tenha especialistas, profissionais de saúde e também idosos como sujeitos, incluídos aí aspectos técnicos e subjetivos na construção de projetos de vida, de projetos de felicidade.

\section{Abstract}

We seek, in this paper, to identify which means healthy alimentation for the elderly and the difficulties found daily to incorporate this set of precepts. We interviewed 202 elderly over 60 years, most females (93.1\%), current or previous participants of

1 Universidade do Estado do Rio de Janeiro. Instituto de Nutrição, Departamento de Nutrição Social. Rio de Janeiro, RJ, Brasil.

2 Universidade do Estado do Rio de Janeiro. Instituto de Nutrição, Programa de Pós-Graduação em Alimentação, Nutrição e Saúde. Núcleo de Estudos sobre Cultura e Alimentação (NECTAR). Rio de Janeiro, RJ, Brasil.

Correspondência / Correspondence

Maria Fátima Garcia de Menezes

Universidade do Estado do Rio de Janeiro

Instituto de Nutrição - Departamento de Nutrição Social

Rua São Francisco Xavier, 524, $12^{\circ}$ andar, Bloco E, sala 12.006

20559-900 Rio de Janeiro, RJ - Brasil

E-mail: m.fatima.menezes@gmail.com

Agradecemos à FAPERJ pelo apoio concedido através do Processo E-26/ 111.058/2008.

Maria Fátima Garcia de Menezes ${ }^{1}$

Elda Lima Tavares'

Débora Martins dos Santos ${ }^{1}$

Carina Loureiro Targueta ${ }^{2}$

Shirley Donizete Prado²

Palavras-chave:

Alimentação. Saúde.

Idoso. Envelhecimento.

Nutrição do Idoso.

Hábitos Alimentares. 
activities at the Open University for Studies on the Elderly of Rio de Janeiro State University (70.8\%). The responses indicate an ideology which has close ties to the concern for health, to prevent or treat chronic and degenerative diseases, with biologicist and medicalizing character of eating distanced from the world of desire and subjectivity. Difficulties concerning purchasing power, family life or solitude, biological issues imposed by the aging process appear as limiting to the regular practice of healthy eating. We believe that, from reflections on these questions, you can enrich the concept of healthy eating, including effectively both inside the health, as disease, considering the importance of a dialogue in which the subjects are experts, health professionals and elderly subjects, included therein, technical and subjective aspects in the construction of life projects, of projects of happiness.
Key words: Feeding. Health. Aged. Aging. Elderly Nutrition. Food Habits.

\section{QUALIDADE DE VIDA, PROMOÇ̃̃O DA SAÚdE E ALIMENTAÇÃO SAUDÁVEL NA VELHICE}

O aumento do tempo de vida é um fenômeno de grande alcance no mundo todo, caracterizado pelo incremento da ordem de 30 anos na esperança de vida de muitos povos nas décadas recentes. Uma verdadeira revolução envolvendo desafios políticos, econômicos, sociais, demográficos e, de modo especial, nos campos da saúde e da alimentação.

No Brasil, peculiaridades devem ser consideradas, uma vez que este nosso país é, historicamente, marcado por fortes desigualdades sociais, por direitos fundamentais (habitação, educação, alimentação, saúde, segurança) ainda longe de serem atendidos, por problemas profundos e complexos com a vasta população jovem, ainda não satisfatoriamente encaminhados. ${ }^{1,2}$

Neste cenário e, em especial, a partir dos anos 1980, discussões importantes passam a ser travadas no Brasil a partir de pesquisas que denunciam a perda do valor social dos velhos, de sua dimensão humana em consequência do desenvolvimento do modelo econômico que reduz o homem à produção e ao consumo puros. ${ }^{3,4,5} \mathrm{~A}$ valorização da cidadania em sua plenitude passa a ocupar a agenda de vários pesquisadores que tomam o envelhecimento como tema de suas investigações. ${ }^{6}$

Estudos de maior abrangência no campo da Saúde Coletiva enfatizam preocupações com a qualidade de vida dos idosos, tanto no que se refere aos seus aspectos subjetivos (bem-estar, felicidade, amor, prazer, realização pessoal), quanto às suas necessidades básicas mais objetivas da vida. ${ }^{7,8}$ Qualidade de vida definida, nas palavras de Minayo, Hartz e Buss ${ }^{9}$ como:

uma noção eminentemente humana, que tem sido aproximada ao grau de satisfação encontrado na vida familiar, amorosa, social e ambiental e à própria estética existencial. Pressupõe a capacidade de efetuar uma síntese cultural de todos os elementos que determinada sociedade considera seu padrão de conforto e bem-estar. $\mathrm{O}$ termo abrange muitos significados, que refletem conhecimentos, experiências e valores de indivíduos e coletividades que a ele se reportam em variadas épocas, espaços e histórias diferentes, sendo portanto uma construção social com a marca da relatividade cultural (p. 8)

Interessa-nos a noção de qualidade de vida que, em sua polossemia, admite discussões, para além das predominantes perspectivas funcionais e medicalizadas da velhice, expressas nas perspectivas muito presentes na ideia de qualidade de vida em saúde. Estudo realizado por Vecchia, Ruiz e Bocchi ${ }^{10}$ sobre o conceito de qualidade de vida entre idosos brasileiros mostra resultados semelhantes a pesquisas internacionais, onde está presente a valorização do bom relacionamento com a família, com amigos e da participação em organizações sociais; da saúde; de hábitos saudáveis; bem-estar, alegria e amor; de condição financeira estável; do trabalho; da espiritualidade; de praticar trabalhos voluntários e de poder aprender mais. O olhar de velhos brasileiros, sua própria concepção de 
qualidade de vida se nos mostram também mais afins a essas perspectivas ampliadas:

Foram identificados três perfis de idosos no município de Botucatu - SP, segundo a definição que eles deram sobre o que era qualidade de vida: o primeiro mencionou situações referentes a relacionamentos interpessoais, equilíbrio emocional e boa saúde, ou seja, é o idoso que prioriza a questão afetiva e a família; o segundo grupo mencionou hábitos saudáveis, lazer e bens materiais, ou seja, é o idoso que prioriza o prazer e o conforto; e o terceiro grupo, que mencionou espiritualidade, trabalho, retidão e caridade, conhecimento e ambientes favoráveis, poderia ser sintetizado como o idoso que identifica como qualidade de vida conseguir colocar em prática o seu ideário de vida (p. 251).

Também, a ideia de promoção da saúde e prevenção de doenças tem estado bastante presente em pesquisas mais recentes dentro da perspectiva de investimentos nas possibilidades de realização humana na velhice. Promoção da saúde é aqui entendida como o processo de capacitação da comunidade para atuar na melhoria da sua qualidade de vida e saúde, incluindo uma maior participação no controle deste processo, o que envolve, necessariamente, políticas públicas saudáveis, criação de ambientes favoráveis à saúde, reforço da ação comunitária, desenvolvimento de habilidades pessoais e reorientação do sistema e dos serviços de saúde. ${ }^{11,12,13}$ Trata-se, então, de uma concepção também mais abrangente de saúde, bastante além da tônica estrita da prevenção e da cura de doenças no espaço ambulatorial e hospitalar, acentuadamente caracterizada pelo uso intensivo de tecnologias de diagnóstico e de intervenção médica que identificam as concepções e práticas voltadas para idoso no mundo moderno.

Devemos aqui considerar as reflexões de Ayres, ${ }^{14}$ cuidando para não enveredar pelos caminhos da polarização entre saúde e doença, mas tomandoos como conceitos diferentes e ao, mesmo tempo, indissociáveis. Nas palavras do autor

O que o enfoque hermenêutico da saúde aqui defendido propõe para a reconstruşão humanizadora das práticas de saúde é que profissionais, serviços, programas e políticas de saúde estejam mais sensíveis e responsivos ao sucesso prático de suas ações, isto é, que orientem a busca de êxito técnico de suas intervenções na direção apontada pelos projetos de felicidade dos destinatários de suas ações. (Grifo do autor, p. 57).

O campo da Alimentação é rico em possibilidades de realização humana quando tomado como lugar em que se estabelecem relações entre seres humanos mediadas pelo alimento, pela comida. ${ }^{15}$ Espaço de interação com a Nutrição - que enfatiza as correspondências entre nutrientes e o corpo biológico normal ou patológico - e com as Ciências dos Alimentos - em seus estudos sobre a composição química dos alimentos, qualidade sanitária e processos de produção. A Alimentação comporta abordagens que vão desde os aspectos relativos a políticas de uso terra e produção, distribuição e comercialização dos alimentos até a escolha coletiva e/ou individual do quê, com quem, onde, como comer, preferências, rejeições, atitudes, práticas alimentares, habitus, ${ }^{16}$ comportamentos plenos de representações, significados, simbolismos. ${ }^{17}$ Comemorações, rituais, desejos, prazeres, cuidados com a saúde, dietas, ideais de beleza corporal, lembranças, finitude; alegrias e tristezas fazem parte, de alguma forma, do universo da Alimentação, que corresponde à noção ampla e potencialmente capaz de abarcar componentes de felicidade, de bem-estar e de segurança presentes no cotidiano de pessoas de todas as idades.

Alimentação, importante campo científico, entretanto, vem sendo pouco trabalhado no mundo da ciência positiva, dada a ênfase histórica - e hegemônica mesmo - da Nutrição em sua identidade com o modelo biomédico de conceber processos de saúde-doença-cuidado. Aqui, a tônica é dada pelo estabelecimento dos limites daquilo que se pode ou não comer em função de patologias comuns a vida moderna, em especial aos velhos, o que fica bem marcado na noção de alimentação saudável veiculada nos anos recentes.

Em maio de 2004, a 57 a Assembleia Mundial da Saúde aprovou a Estratégia Global da Organização Mundial da Saúde (OMS) sobre Alimentação Saudável, Atividade Física e Saúde. ${ }^{18}$ $\mathrm{O}$ discurso enunciado pela OMS no documento 
oficial que lança a "Estratégia Global" considera o crescente peso que representam as doenças não transmissíveis - principalmente as cardiovasculares, o diabetes tipo 2 e determinados tipos de câncer - o perfil de morbi-mortalidade mundial e a ideia de que a prevenção dessas doenças constitui um desafio muito importante para a saúde pública mundial. Os fatores de risco mais significativos para essas patologias são, segundo a OMS, a hipertensão arterial, a hipercolesterolemia, a insuficiente ingestão de frutas, verduras e legumes, a falta de atividade física e o consumo do tabaco - cinco desses fatores de risco estão estreitamente associados à alimentação pouco saudável e à falta de atividade física. Um enfoque integrado das causas da má alimentação e da diminuição da atividade física contribuiria para reduzir a intensidade dessas doenças no futuro.

Garcia $^{19}$ discute essa abordagem sobre o conceito de alimentação saudável destacando sua forte associação à visão biológica/metabólica, pautado nas recomendações científicas sobre ingestão de nutrientes necessários à vida e saúde. A Organização Mundial de Saúde recomenda que os países adotem estratégias que visem a alcançar ingestão de nutrientes "dentro dos limites aconselhados". As recomendações incluem (p.9):

- lograr un equilibrio energético y un peso normal;

- limitar la ingesta energética procedente de las grasas, sustituir las grasas saturadas por grasas in-saturadas y tratar de eliminar los ácidos grasos trans;

- aumentar el consumo de frutas y hortalizas, así como de legumbres, cereales integrales y frutos secos;

- limitar la ingesta de azúcares libres;

- limitar la ingesta de sal (sodio) de toda procedencia y consumir sal yodada

Nas palavras da autora, ${ }^{19}$ "dieta é o termo que melhor traduz o enfoque atual de Alimentação saudável” (p.14) - ou seja, a associação da alimentação com a prevenção de doenças crônicodegenerativas confere ao ato de comer um caráter de medicalização. Isso fica bem expresso quando a alimentação é identificada como causadora das doenças e a sua composição química é hipervalorizada, como nos diz Lifschitz: ${ }^{20}$

O rótulo do alimento, indicando sua composição, transforma-se, assim, no equivalente a uma bula ("indicações de uso e contra-indicações”), e o alimento, desagregado em componentes e funções, em medicamento, e, enquanto tal, sujeito à fórmula "serve para...". (p. 77)

São abordagens centradas em premissas biologicistas, tecnicistas, intervencionistas que ignoram - ou, quando muito, mencionam sem efetivamente valorizar - os aspectos sociais e culturais tão fortemente presentes na Alimentação. Parece que se fala de Nutrição e não de Alimentação.

Retomando as perspectivas de Ayres ${ }^{14}$ sobre saúde e doença, devemos aqui cuidar também para não investir na polarização entre Alimentação e Nutrição - menos ainda com se fora um saúde e outro doença-e, sim, buscando as trajetórias que consideram suas diferenças e indissociabilidade na vida cotidiana. Não nos interessa enveredar pela dicotomia que hoje parece se colocar nos debates sobre alimentação saudável tomada como o lugar do confronto entre o ponto de vista biológico, de caráter normativo e disciplinador e as perspectivas mais situadas no campo da cultura, das subjetividades, de caráter simbólico e social. Está no nosso foco buscar rumos que nos orientem para os lugares em que as tentativas de êxitos técnicos da razão instrumental que caracteriza profissionais de saúde e especialistas possam estar em encontro dialógico com os movimentos no sentido de sucessos práticos que identificam os idosos em suas ações cotidianas de construção de projetos de felicidade no que se refere à Alimentação, à Nutrição e aos Alimentos.

Procuramos, neste artigo, identificar o que significa alimentação saudável para pessoas idosas $\mathrm{e}$ as dificuldades encontradas no cotidiano para a incorporação desse conjunto de preceitos. Talvez, a partir de reflexões sobre essas questões, seja possível enriquecer a noção de alimentação saudável, incluindo efetivamente em seu interior tanto a saúde, quanto a doença, considerando a importância de um diálogo que tenha 
especialistas, profissionais de saúde e também idosos como sujeitos de seus projetos de vida, incluídos aí aspectos técnicos e subjetivos na construção de projetos de vida, de projetos de felicidade.

\section{PERCURSO TEÓRICO-METODOLÓGICO}

A Universidade do Estado do Rio de Janeiro (UERJ) realiza anualmente um evento aberto a toda a comunidade com vistas a divulgar suas atividades denominado UERJ SEM MUROS. Nos anos de 1999 a 2001 entrevistamos todos os idosos que procuraram espontaneamente o stand do nosso Projeto de Extensão Nutrição e Terceira Idade (PNTI) no evento. Adotamos um questionário padronizado com alguns itens fechados - abordando sexo, idade, escolaridade e moradia - e outros mais abertos - sobre significados de alimentação saudável e eventuais dificuldades para manter esse padrão alimentar. Os entrevistadores, alunos do Curso de Graduação em Nutrição, bolsistas do PNTI e estagiários, foram treinados previamente para possibilitar ao entrevistado liberdade de expressão e para transcrever as respostas.

Consideramos, para este estudo, a utilização de entrevistas entendidas como "conversa com finalidade", ${ }^{21}$ possibilitando ao entrevistado espaço mais aberto para suas considerações sobre o tema abordado. Tomamos a análise temática como caminho principal para discutir significados presentes nas falas dos idosos entrevistados. ${ }^{21}$

Participaram 202 idosos com mais de 60 anos, a maioria do sexo feminino $(93,1 \%)$ e frequentadores atuais ou pregressos de atividades da UnATI $(70,8 \%)$.

A pesquisa foi conduzida dentro de padrões éticos exigidos pela Comissão Nacional de Ética em Pesquisa do Conselho Nacional de Saúde do Ministério da Saúde em conformidade com o disposto na Resolução CONEP no 196/96 e encontra-se amparada pelo parecer número 0293/ 2005 do Comitê de Ética da UERJ.
ALIMENTAÇ̃̃O SAUDÁVEL: sobre normas instrumentais e projetos de vida e de felicidade

Entre os participantes $69,8 \%$ referiram o recebimento anterior de orientação nutricional. São, portanto, pessoas que trazem certa bagagem de informações nutricionais e que as expressaram de modo bastante enfático em várias passagens das entrevistas.

A noção de alimentação saudável estrutura-se, para os idosos entrevistados, na polarização entre alimentos "bons" e "maus" para a saúde. Entre os primeiros encontram-se as "frutas", "verduras", "legumes" e "carnes brancas". Foram utilizados termos como "rica em", "máximo de", "com muito" para se referir a esses alimentos - são expressões que evidenciam a ênfase dada a estes alimentos. Os entrevistados valorizaram as vitaminas, minerais, proteínas e fibras. Algumas falas dos entrevistados que demonstram tal representação:

$$
\begin{aligned}
& \text { "Legumes de montão!”(E7) } \\
& \text { "Toda enriquecida com bastante verduras e legumes"(E55) } \\
& \text { "Maisfrutas, verduras, legumes, carne branca”(E13) } \\
& \text { "Valorizar saladas e vegetais" (E16) }
\end{aligned}
$$

Por outro lado, termos como evitar, não entra, sem, pouco, o minimo de, pobre em, foram associados ao consumo de "frituras", "carnes vermelhas", "doces", "massas" e "sal". Calorias, óleos, gorduras e carboidratos, açúcar e sal apresentaram-se como verdadeiros "vilões" quando se pretende viver com sem doenças ou tendo-as controladas. Algumas falas ilustram essa perspectiva:

$$
\begin{aligned}
& \text { "Sem gordura e fritura"(E10) } \\
& \text { "Aquela que não entra gordura"(E51) } \\
& \text { "Pouco carboidrato, evitar açúcar"(E12) }
\end{aligned}
$$

Esses dados coincidem com os encontrados por Garcia. ${ }^{22}$ Em seu estudo, com indivíduos adultos funcionários públicos, nos diz a autora

No que diz respeito à qualificação da dieta, as gorduras, indistintamente saturadas e 
insaturadas e o colesterol são considerados os principais vilões da Alimentação prejudicial à saúde. Considera-se como prática alimentar "saudável" comer mais vegetais e frutas. O arroz e feijão aparecem como base da dieta, mas não entram como parte do discurso do que seria "saudável", aparecem muito vagamente com caráter negativo, quando sob a égide da saúde (p. 63).

Também foram evidenciadas nas entrevistas falas que incorporaram o "comer de tudo com moderação", "reine todos os nutrientes", "tem que ser balanceada".

Uma leitura poderia nos dizer de regras gerais rígidas, inflexíveis, onde não deve haver lugar para alimentos maus que levam a doenças e somente os bons, ou seja, os que não causam doenças, devem ser ingeridos. Aqui podemos trazer à baila as muito usadas listas de alimentos proibidos e permitidos tão presentes nos consultórios médicos e de nutricionistas, como em palestras, matérias educativos distribuídos em eventos associados a prevenção de doenças ou, ainda, em revistas e programas de rádio e televisão quando lidam com a temática aqui em questão.

Trata-se de um ideário que apresenta estreita vinculação à preocupação com a saúde, no sentido de prevenir ou tratar as doenças crônicodegenerativas, com caráter biologicista e medicalizador do ato de comer. O simbolismo mais presente é a restrição e o inatingível. Como afirma Garcia 19 “... o que norteia a concepção de Alimentação saudável atual é aquilo que ela pode eventualmente evitar."

Tal premissa se sustenta nas recomendações científicas e na competência do profissional, que define o que é certo ou errado. Como pode ser observado nos trechos a seguir:

A educação nutricional visa à melhoria da saúde pela promoção de hábitos adequados, eliminação de práticas dietéticas insatisfatórias, introdução de melhores práticas higiênicas e uso mais eficiente dos recursos alimentares (p.18). ${ }^{23}$

A educação nutricional, por sua vez, incentiva o consumo de alimentos naturais, frutas, hortaliças e recomenda evitar as guloseimas, as gorduras saturadas e os alimentos artificiais (p. 18). ${ }^{23}$

Sob a égide nutricional dos proibidos e permitidos, a alimentação fica restrita a um mundo de erros e acertos, perdendo em perspectivas de viabilidade social e de sua riqueza cultural, além de distanciada do mundo dos desejos e da subjetividade. Resulta, ao significar prevenção ou tratamento de doença, em sentido controlador de riscos inexoráveis de incapacidade, dependência e morte. Errar aqui é, nos termos colocados pelo modelo nutricional biomédico, reduzir o tempo de vida independente.

O mundo idealizado da racionalidade nutricional encontra-se largamente disseminado entre idosos que frequentam serviços de saúde, um dos lugares onde entram em contato com as informações sobre alimentação saudável concebidas a partir desse paradigma biomédico. A se tomar por base as falas dos idosos entrevistados neste estudo, alimentação saudável corresponderia a comer diariamente peixe ou frango magros cozidos, grelhados ou assados acompanhados de legumes cozidos em água (e tudo com pouco ou nenhum sal), verduras e frutas. O modelo nutricional idealizado, onde as dificuldades para manter a alimentação saudável em suas regras de alimentos bons e maus fazem com que o número de anos de vida sem doença apareça distanciado da visão ampliada da saúde, que incorpora a garantia de prazer e felicidade ao alimentar-se. . $^{1920,22,24}$

Tentando ir um além do ideal da nutrição balanceada ou equilibrada construída sobre o pilar da moderação na ingestão dos alimentos permitidos - uma vez que os proibidos não encontram lugar nesse terreno árido - procuramos explorar percepções de idosos perguntando sobre eventuais dificuldades que encontrariam para a prática de uma alimentação saudável. Na esfera socioeconômica aparecem os recursos financeiros, ilustrado nas falas a seguir:

"Comprar os alimentos... a familia é grande" (E7)

'Dinheiro, porque às vezes o dinheiro não dápara comprar o que é saudável" (E24) 
E também a estrutura familiar é relatada como um fator importante. De um lado, viver com filhos, netos e marido envolve lidar e administrar diferentes necessidades e gostos; de outro, a desmotivação do comer só:

\section{"Problemas familiares, gostos distintos"(E84) \\ "Moro sozinha, que tira a motivação para faz̧er comida"(E91)}

Também em relação a este aspecto houve vários relatos que apontam para a estrutura social e a nova organização da alimentação: o comer na rua, nas festas, na casa de parentes. As "preferências" e "gostos" também devem ser considerados como uma dificuldade. Garcia ${ }^{19}$ entende que as práticas são marcadas pela ambiguidade e conflito entre a preocupação com a saúde e o atendimento ao desejo/paladar individual (1997). Pode-se observar nas falas a seguir:

\section{"Como o que eu tenho vontade"(E16) \\ "Não gosto de couve, brócolis, couve-flor"(E3) \\ "Gosto mais de massas, frituras"(E61)}

Um último tópico, e não menos importante, refere-se às limitações biológicas impostas pelo processo de envelhecimento. Dificuldades de locomoção, interferindo diretamente na compra dos alimentos, redução de paladar e alterações digestivas foram citadas.

Esse conjunto de dificuldades para manter o ideal nutricional ancorado no paradigma biomédico e apresentado pelos idosos como "alimentação saudável" nos coloca mais próximos das suas experiências de vida e, talvez, de uma ampla parcela da população. Longe de se mostrarem obstáculos, para nós, correspondem a nortes importantes que podem nos auxiliar nas reflexões sobre qualidade de vida, promoção da saúde e projetos de viver bem.

Essas dificuldades nos dizem da necessidade de identificar novos modos de operar no cotidiano do cuidado, enfatizando que, quando se trata de idosos, alguma(s) doença(s) geralmente está(ão) presente(s). Falam da necessidade de entender o indivíduo nas suas relações, na sua vida, seus diferentes rituais alimentares e mudanças nas práticas proporcionadas pela sua inserção em diferentes grupos. E ainda a tradição, a infância, a história vivida, as quais deixam marcas importantes na nossa memória alimentar. ${ }^{25}$ Tudo isso em meio a hipertensões, diabetes, cardiopatias, obesidades, medo de ficar dependendo de familiares, medo de comer alimentos contaminados por agrotóxicos, cheios de hormônios, corantes, prazos de validade duvidoso... Medo de morrer já que, entre idosos, a finitude é questão cada vez mais presente com o passar do tempo.

Quais seriam os projetos de vida e de felicidade de pessoas idosas e como o comer participa desse devir? Como pensar uma alimentação que considere os conhecimentos instrumentais presentes nos parâmetros nutricionais e que ao mesmo tempo venha a dar conta de planos de bem viver, aos projetos de felicidade aos 63, 87, 94 ou 105 anos? Como encaminhar ações em que questões alimentares e nutricionais devam ser tratadas buscando-se compreender sua complexidade no cotidiano dos idosos, sem perder vista a busca por qualidade de vida, promoção da saúde, tratamento de doenças e os projetos de boa vida e de felicidade desses sujeitos e dos profissionais de saúde e especialistas?

Questões complexas para as quais, seguindo os caminhos indicados em Ayres, nos aventuramos a apresentar algumas proposições. Um eixo central para desenhos nesse campo corresponde ao diálogo. Nas palavras do autor:

Evidentemente não nos referimos aqui ao diálogo como mero recurso para obtenção de informaçōes requeridas pelo manejo instrumental do adoecimento, forma de produ₹ir uma narrativa estruturada segundo esse interesse, que é o modelo típico da anamnese clássica. O sentido forte de diálogo na perspectiva hermenêutica é o de fusão de borizontes (GADAMER, 2004), isto é, de produção de compartilhamentos, de familiarização e apropriação mútua do que até então nos era desconbecido no outro, ou apenas supostamente conhecido. Não basta, nesse caso, apenas fazer o outro falar sobre aquilo que eu, profissional de saúde, sei 
que é relevante saber. É preciso também ouvir o que o outro, que demanda o cuidado, mostra serindispensável que ambos saibamos para que possamos colocar os recursos técnicos existentes a serviço dos sucessos práticos almejados (Grifo do autor, p. 58).

Por esse caminho, podemos considerar a possibilidade de perguntar ao idoso, por exemplo: "O que acha de sua alimentação nessa etapa de vida e diante de sua condição de saúde?" ou "O que pensa que posso fazer para ajudá-lo em sua alimentação?”. Ao proceder dessaforma mais aberta, mais disponível a ouvir e a trocar, o profissional pode estar operando de modo a possibilitar a fusão entre racionalidade instrumental e perspectivas práticas, num movimento de superação das dificuldades enfrentadas, ali colocadas e recolocadas a nu, a cada encontro, para ambos, como um desafio, um projeto em permanente re-elaboração.

\section{REFERÊNCIAS}

1. Camarano AA, organizador. Muito além dos 60: os novos idosos brasileiros. Rio de Janeiro: IPEA; 1999.

2. Veras RP, Caldas PC. Promovendo a saúde e a cidadania do idoso: o movimento das universidades da terceira idade. Ciên Saúde Colet 2004: 9(2):423-32.

3. Beauvoir S. A velhice. Rio de Janeiro: Nova Fronteira; 1970.

4. Haddad EGM. A ideologia da velhice. São Paulo: Cortez; 1986.

5. Bosi E. Memória e sociedade: lembranças de velhos. São Paulo: T. A. Queiroz-Edusp; 1987

6. Assis M, Hartz ZMA, Valla VV. Programas de promoção da saúde do idoso: uma revisão da literatura científica no período de 1990 a 2002. Ciên Saúde Colet 2004 set; 9(3): 557-81.

7. Neri AL, organizador Qualidade de vida na velhice: enfoque multidisciplinar. Campinas: Alinea, 2007.300 p.

8. Diogo MJD, Neri AL, Cachioni M, organizadores. Saúde e qualidade de vida na velhice. 1. ed. Campinas: Alínea; 2003. v. 1.236 p.

9. Minayo Maria C SHartz Zulmira Maria de Araújo, Buss Paulo Marchiori. Qualidade de vida e saúde: um debate necessário. Ciên Saúde Colet 2000; 5(1): 7-18.
Nesse processo, olhares atentos, gestos que acolhem, ações que denotam respeito e consideração são absolutamente inovadores, posturas que demonstram conhecimento técnico e, ao mesmo tempo, disponibilidade para adaptações a culturas diferentes, desejos, limitações identificadas podem propiciar o encontro de subjetividades e o delineamento de projetos de vida e de felicidade para ambas as partes envolvidas, além de outras partes com cuidadores que devem ter lugar assegurado, tanto quanto profissionais ou usuários idosos de serviços de saúde.

Com outras características mais pertinentes, o mesmo pode ser pensado para ações que envolvem populações de idosos que vivem em instituições asilares ou que se destinem a grupos populacionais outros.

10. Dalla Vecchia R, Ruiz T, Bocchi SCM, Corrente JE. Qualidade de vida na terceira idade: um conceito subjetivo. Revista brasileira de epidemiologia 2005 set; 8(3): 246-52.

11. Ferreira JR, Buss PM. O que o desenvolvimento local tem a ver com a promoção da saúde. In: Zancan L, Bodstein $\mathrm{R}$, Marcondes WB, organizadores. Promoção da saúde como caminho para o desenvolvimento local - a experiência em Manguinhos - RJ. Rio de Janeiro: Abrasco/ Fiocruz; 2002. p. 15-38.

12. Adriano JR, Werneck GAF, Santos MA, Souza RC. A construção de cidades saudáveis: uma estratégia viável para a melhora da qualidade de vida? Ciên Saúde Colet 2000; 5(1):53-62.

13. Paim JS, Almeida Filho N. A crise da saúde pública e a utopia da saúde coletiva. Instituto de Saúde Coletiva/ Universidade Federal da Bahia, Salvador; 2000.

14. Ayres JRCM. Uma concepção hermenêutica de saúde. Physis 2007:17(1):43-62.

15. Prado SD. A pesquisa sobre Alimentos, Alimentação e Nutrição no Brasil: reflexões sobre a produção de conhecimento e saberes. Projeto de pesquisa. Rio de Janeiro: Instituto de Nutrição da Universidade do Estado do Rio de Janeiro, 2005. 
16. Bourdieu P. O poder simbólico. 7 ed. Rio de Janeiro: Bertrand; 2004.

17. Fischler C. Gastro-nomia y gastro-anomia. Sabiduría del cuerpo y crisis biocultural de la alimentación contemporânea. In: Contreteras JH, organizador. Alimentación y cultura: necessidades, gustos y costumbres. Barcelona: Universitat de Barcelona Publicacions, 1995,p. 9-26.

18. Organización Mundial de la Salud. Estrategia mundial sobre régimen alimentario, actividad física y salud. Ginebra: OMS; 2004. Disponível em http://www.who.int/dietphysicalactivity/ strategy/eb11344/strategy_spanish_web.pdf. Acesso em 22 abr. 2009.

19. Garcia RWD. Representações sociais da alimentação e saúde e suas repercussões no comportamento alimentar. Physis 1997; 7(2): 51-68.
20. Lifschitz J. Alimentação e cultura: em tomo ao natural. Physis 1997 dec; 7(2).

21. Minayo MCS. O desafio do conhecimento. 6. ed. São Paulo: Hucitec, 2000.

22. Garcia RWD. Práticas e comportamento alimentar no meio urbano: um estudo no centro da cidade de São Paulo. Cad Saude Publica 1997; 13(2): 455-67.

23. Motta DG, Boog MCF. Educação Nutricional. 2. ed.São Paulo: IBRASA, 1987.

24. Boog MCF. Educação Nutricional em serviços de saúde. Cad Saúde Pública 1999; 15 (sup.2):139-47.

25. Mintz SW. Comida e antropologia: uma breve revisão. Revista Brasileira de Ciências Sociais 2001 out; $16(47)$. 
